\title{
Perception towards research among undergraduate physical therapy students
}

\begin{abstract}
Objective: To know the perception of students regarding Research among undergraduate physical therapy students.

Material and methods: This was an observational cross sectional study with nonprobability convenience sampling technique. The data was collected from different physical therapy institutes, Isra Institute of Rehabilitation sciences Karachi and Isra University Hyderabad. The duration of the study was July to December 2014. Inclusion criteria were Final year undergraduate Physical Therapy Students, either gender and who were willing to participate. Exclusion criteria were Undergraduate students of $1^{\text {st }} 2^{\text {nd }}, 3^{\text {rd }}$ and $4^{\text {th }}$ year, Post graduate physical therapy students and those who did not give consent. Data Collection Procedure was a questionnaire with consent form was distributed to all participants. Validated questionnaire adapted from Andrea Tschirner et al study in 2011. Questionnaire contained closed ended questions. SPSS version 20.0 was used to analyzed the data.
\end{abstract}

Results: A total of 100 participants involved in the study. There were 72 female and 28 male participants. There were $84 \%$ respondents strongly agreed that research in under graduate is necessary for positive growth in the physiotherapy profession, $12 \%$ (12) participants were agreed and $4 \%$ (4) was disagreed. When the participants were asked that is research exploring the effectiveness of physiotherapy treatment is necessary for positive growth in the physiotherapy profession maximum number of individuals showed strongly agree response i.e. $75 \%$ (75) however $25(25 \%)$ participant marked agree option.

Conclusion: Majority of the students perceive that research in undergraduate is necessary for positive of the physical therapy profession. Scientific research in postgraduate is necessary for positive growth in the physiotherapy profession.

Keywords: perception, research, physical therapy, patient, growth
Volume 7 Issue 3 - 2018

\author{
Abida Arif,' Muhammad Arif Siddiqui, ${ }^{2}$ \\ Khalid Aziz,' Ghousia Shahid,' Asif Shiekh, ${ }^{3}$ \\ Muhammad Faisal Fahim' \\ 'Bahria University Medical \& Dental College, Pakistan \\ Institute of Physical Medicine \& Rehabilitation (IPMR), Dow \\ University of Health Sciences, Pakistan \\ ${ }^{3}$ Isra Institute of Rehabilitation Sciences, Pakistan
}

Correspondence: Muhammad Faisal Fahim, Researcher, Bahria University Medical \& Dental College, Karachi, Pakistan, Email faisalfahim88@hotmail.com

Received: March 28, 2018| Published: May 08, 2018

\section{Introduction}

Professional research is the foundation of advancement in technology and medicine. With the consistent growth and variety of professions within Physical Therapy, it is important for medical imaging professionals to stay current on technological advances, as well as comprehending and generating additional research in their area of practice. Growth of their practice will enhance the level of patient care. Thus, the strength of physical therapy depends on research to expand its scientific basis for practice and patient care, leading to research-based practice or clinically oriented practice, a vision advocated by the American Physical Therapy Association (APTA) involved in research among physical therapists. ${ }^{1}$ The most commonly reported barriers to physical therapy research, however, were lack of time and funding, a high work load, and unfamiliarity with research procedures and statistics. ${ }^{2}$ Over the years, medical education has evolved from learning facts to acquiring skills, knowledge and attitude. ${ }^{3}$ This makes it very important to promote critical thinking and reasoning skills in medical students at an earlier age in order to develop a positive attitude towards health care research. ${ }^{4}$ Research motivation is influenced by previous educational background, research experience and also possibly culture and gender. Similar findings were reported in Pakistani studies. ${ }^{5}$ Postulated explanations for the decline of the physician-scientist include less financial incentive, family, practice philosophy, lack of research training and inadequate exposure to research before career paths are determined, but the primary reason is the development of research appetite at an earlier stage in the student. ${ }^{6}$ Clinical instructors play a vital role in the clinical learning of undergraduate physical therapy students. It is very important for the clinical instructors to train the physical therapy students in accordance with the growing challenges. The clinical learning must prepare the students to be able to work as primary health care providers. ${ }^{7}$ Clinical instructor must use the best possible learning strategies and learning environments in order to ensure the development of good clinical reasoning in the students. Significant mismatch has been found between the preferred and actual learning environments of paramedical students. ${ }^{8}$ The bridge version of clinical education organization is in use in British common wealth countries including Nigeria, in which clinical rotations are organized in hospitals that are affiliated with respective universities of the students. ${ }^{9}$ The same bridge version is commonly used in Pakistan in most of the physical therapy institutes. The characteristics of an ideal clinical teacher as perceived by the students have been reported to be professionalism, being a role model for the students, describing the decision making process, considering the self-respect of students, willingly helping the students, appropriate knowledge of the respective subject and ability to demonstrate and convey that knowledge to the students. ${ }^{10}$ Various factors are important in the clinical education of the undergraduate physical therapy students and a good clinical instructor 
can be regarded as the integral part of effective clinical learning. ${ }^{11}$ Various studies have been conducted to find out the perception of students regarding their clinical instructors in order to improve the process of clinical education and to maximize the benefits students get from the clinical learning. ${ }^{12}$ Evidence-based practice (EBP), research utilization (RU), and knowledge translation (KT) are interrelated concepts that pertain to the identification, utilization and application of knowledge from research sources to clinical practice. EBP has been defined as "the integration of clinical expertise, patient values, and the best research evidence into the decision making process for patient care". RU is a sub-set of EBP, which refers to "that process by which specific research-based knowledge is implemented in practice". ${ }^{13} \mathrm{KT}$, on the other hand, emphasizes the synthesis, dissemination, exchange and application of knowledge from research findings, and from other sources, to influence changes in practice and improve health outcomes. ${ }^{14}$ Research is necessary to advance the practice of physical therapy in order to assist physical therapists in the development and testing of knowledge unique to their practice. ${ }^{15}$ This study aims to know the perception of students regarding Research among undergraduate physical therapy students.

\section{Materials and methods}

This was an observational cross sectional study with nonprobability convenience sampling technique. The data was collected from different physical therapy institutes, Isra Institute of Rehabilitation sciences Karachi and Isra University Hyderabad. The duration of the study was July to December 2014. One hundred undergraduate physical therapy students participated in the study. Inclusion criteria were Final year undergraduate Physical Therapy Students, either gender and who were willing to participate. Exclusion criteria were Undergraduate students of $1^{\text {st }} 2^{\text {nd }}, 3^{\text {rd }}$ and $4^{\text {th }}$ year, Post graduate physical therapy students and those who did not give consent. Data Collection Procedure was a questionnaire with consent form was distributed to all participants. Validated questionnaire adapted from
Andrea Tschirner et al study in 2011. Questionnaire contained closed ended questions.

\section{Statistical analysis}

Data was entered and analyzed through the software statistical package for social sciences (SSPS) version 20.0. Descriptive Statistics Frequency, percentage was used for analysis. Bar and Pie chart were also used foe the presentation of data.

\section{Results}

The response of the participants was 100\%. All participants took an interest in the study and showed their concern. They answered all questions in the questionnaire. A total of 100 participants involved in the study. There were 72 female and 28 male participants. There were $84 \%$ (84) participants strongly agreed that research in under graduate is necessary for positive growth in the physiotherapy profession, $12 \%$ (12) participants were agreed and 4\% (4) was disagreed. When the participants were asked that is research exploring the effectiveness of physiotherapy treatment is necessary for positive growth in the physiotherapy profession maximum number of individuals showed strongly agree response i.e. $75 \%$ (75) however 25(25\%) participant marked agree option. In the question related to research exploring the benefits of physiotherapy treatment is necessary for positive growth in the physiotherapy profession; maximum number of participants i.e.68 (68\%) said they strongly agreed to it, $32(32 \%)$ of individuals replied they just agreed. In the question related to research exploring the cost versus benefits ratio of physiotherapy treatment is necessary for positive growth in the physiotherapy profession. Participant's responses were as followed: $63 \%$ (63) participants replied strongly agreed, $37 \%$ (37) individual replied agreed. In the question related to research exploring the safety of physiotherapy treatment is necessary for positive growth in the physiotherapy profession. Participant's response was as followed: $70 \%$ (70) participants replied strongly agreed, 30\% agreed and none in disagree (Figures 1-6) (Table 1).

Table I Questions regarding Research understandings related to Physical Therapy

\begin{tabular}{|c|c|c|c|c|c|}
\hline Questions & $\begin{array}{l}\text { Strongly } \\
\text { Agree }\end{array}$ & Agree & Neutral & Disagree & $\begin{array}{l}\text { Strongly } \\
\text { Disagree }\end{array}$ \\
\hline Research in undergraduate is necessary for positive growth in the physiotherapy profession. & $84 \%$ & $12 \%$ & $0 \%$ & $4 \%$ & $0 \%$ \\
\hline $\begin{array}{l}\text { Research exploring the effectiveness of physiotherapy treatment is necessary for positive } \\
\text { growth in the physiotherapy profession. }\end{array}$ & $75 \%$ & $25 \%$ & $0 \%$ & $0 \%$ & $0 \%$ \\
\hline $\begin{array}{l}\text { Research exploring the benefits of physical therapy treatment is necessary for positive } \\
\text { growth in the physiotherapy profession }\end{array}$ & $68 \%$ & $32 \%$ & $0 \%$ & $0 \%$ & $0 \%$ \\
\hline $\begin{array}{l}\text { Scientific research exploring the cost versus benefits ratio of physiotherapy treatment is } \\
\text { necessary for positive growth in the physiotherapy profession. }\end{array}$ & $72 \%$ & $28 \%$ & $0 \%$ & $0 \%$ & $0 \%$ \\
\hline $\begin{array}{l}\text { Research exploring the safety of physiotherapy treatment is necessary for positive growth } \\
\text { in the physiotherapy profession. }\end{array}$ & $70 \%$ & $30 \%$ & $0 \%$ & $0 \%$ & $0 \%$ \\
\hline $\begin{array}{l}\text { Scientific research exploring the mechanisms of physiotherapy treatment is necessary for } \\
\text { positive growth in the physical therapy profession }\end{array}$ & $63 \%$ & $37 \%$ & $0 \%$ & $0 \%$ & $0 \%$ \\
\hline $\begin{array}{l}\text { Physiotherapists with research backgrounds are the most qualified to conduct studies with } \\
\text { regard to physiotherapy treatment. }\end{array}$ & $68 \%$ & $29 \%$ & $0 \%$ & $3 \%$ & $0 \%$ \\
\hline A demand exists for researchers in the physiotherapy profession & $64 \%$ & $32 \%$ & $0 \%$ & $4 \%$ & $0 \%$ \\
\hline $\begin{array}{l}\text { Research will result in greater validation of physiotherapy treatment among the health care } \\
\text { professions }\end{array}$ & $76 \%$ & $24 \%$ & $0 \%$ & $0 \%$ & $0 \%$ \\
\hline $\begin{array}{l}\text { Do you plan to have participated in research activities before you finish the physical } \\
\text { therapy program? }\end{array}$ & $75 \%$ & $25 \%$ & $0 \%$ & $0 \%$ & $0 \%$ \\
\hline I am very interested on conducting basic science research. & $85 \%$ & $10 \%$ & $0 \%$ & $5 \%$ & $0 \%$ \\
\hline I am very interested on conducting clinical research. & $65 \%$ & $35 \%$ & $0 \%$ & $0 \%$ & $0 \%$ \\
\hline
\end{tabular}




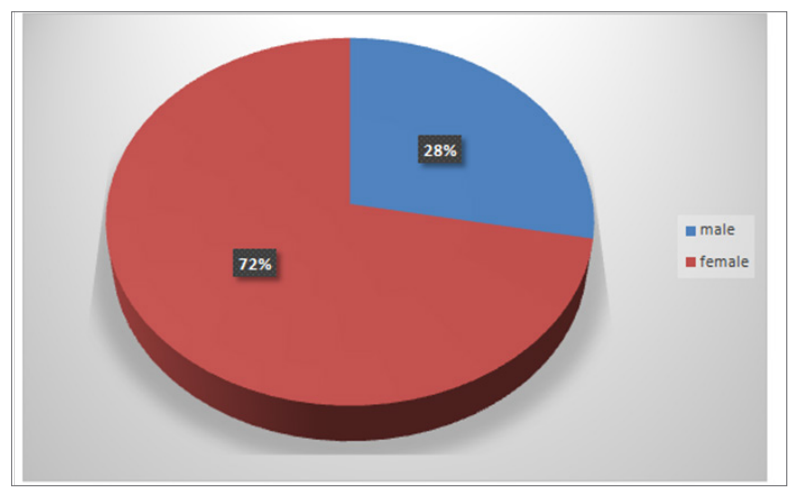

Figure I Gender distribution.

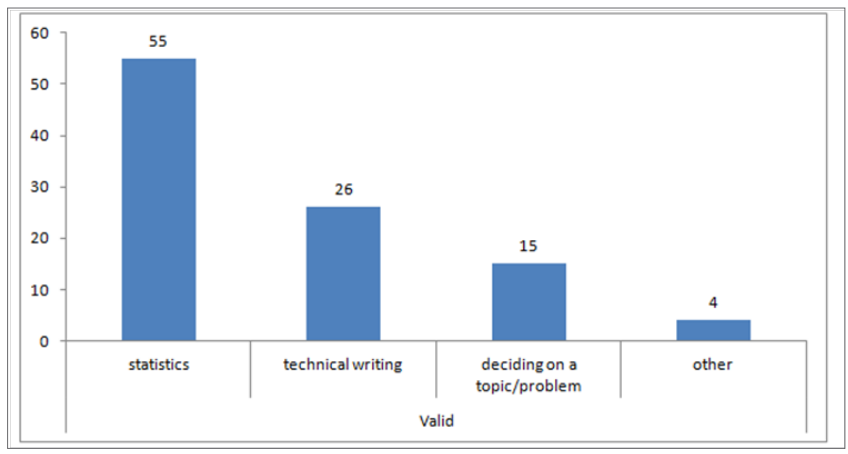

Figure 3 What aspect of research do you find the most challenging?

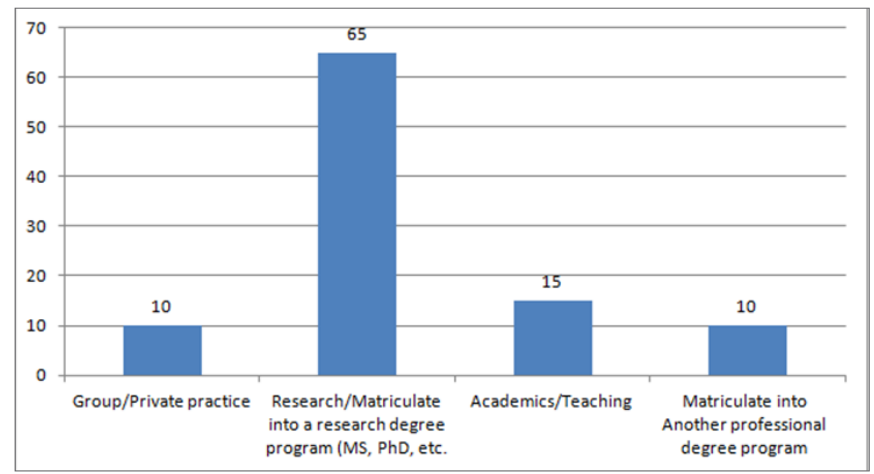

Figure 5 What are your plans after graduation from Physiotherapy college/ institute?

\section{Discussion}

This study identifies the perception of undergraduate physical therapy students towards research. And to identify the barriers towards perceptions in research. The research culture and barriers faced by the physical therapy students towards participation in research. There was an acceptable response rate to this self-administered questionnaire. Data analysis reveals that $86 \%$ (86) participants strongly agreed that scientific research in graduate is necessary for positive growth in the physiotherapy profession. 9\% (9) participants were agreed and 1\% (1) was disagreed and only $3 \%$ (3) individuals showed neutral response.

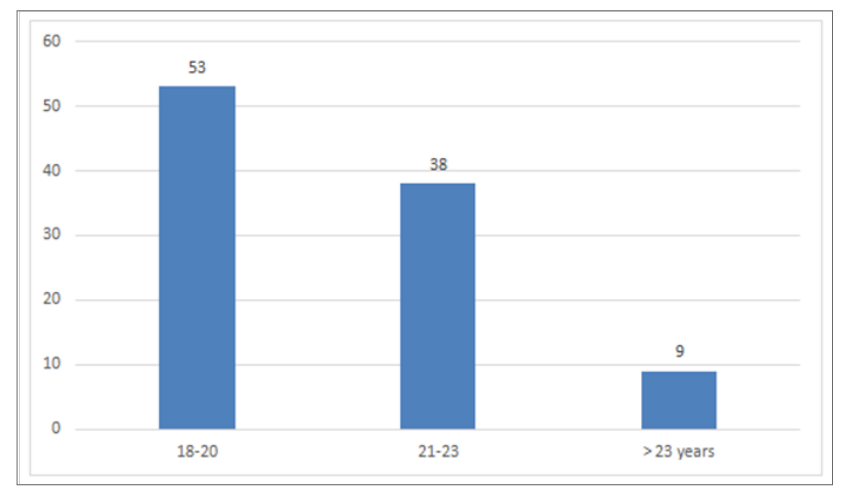

Figure 2 Age Group (years).

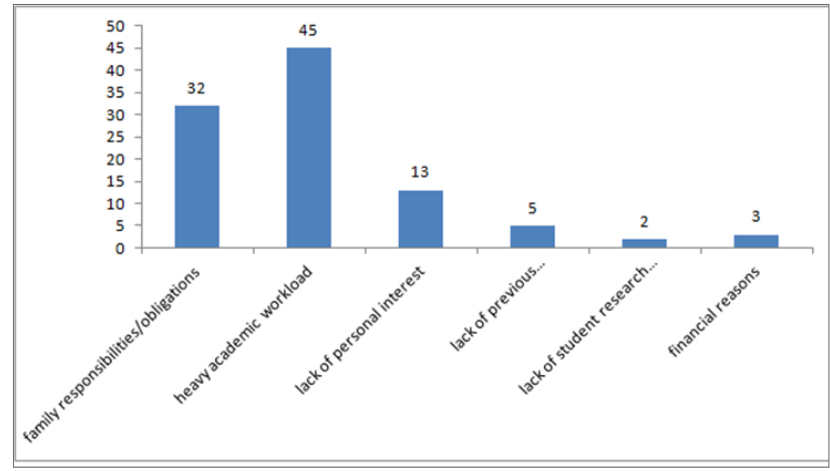

Figure 4 What is your greatest deterrent to being more involved in research?

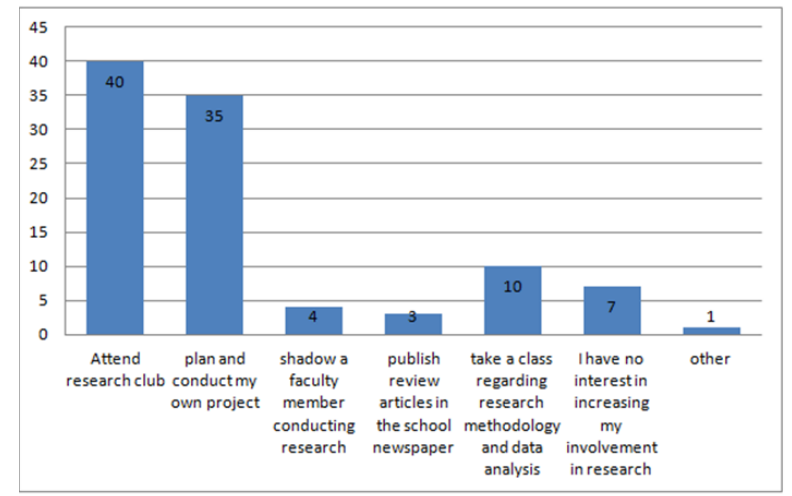

Figure 6 What are your plans after post-graduation from Physiotherapy college/institute?

A research study conducted by Kamwendo ${ }^{16}$ concluded that how research activities and perceptions enhancing knowledge of physical therapy students. The outcomes found participants had a positive attitude towards research, mainly for the activity to review the literature to keep the knowledge update and use research results to improve practice. Bendixen et al. ${ }^{17}$ reported that research related activities considered to be an important part of occupational therapists growth of the professional role and status. Common barriers included by participants in research related activities such as more workload and lack of time. Similarly our study also talks about the research barriers among the physical therapy students. It shows more than talking. It shows that the students needed to read physiotherapists or other health 
care related professional research papers. They also advise others to take a course that focused on experimental design and/ research methodology. This study as compared with Karlsson ${ }^{18}$ reports that student research related activities considered to be an important part of their growth of the professional role and status. To read the research literature for current knowledge was the more important for research activity and the second most essential activity was applying research outcomes to improve physical therapy practice. Common barriers included by participants in research related activities such as more workload and lack of time.

Siamian et al. ${ }^{19}$ reported that participants had a positive attitude towards research, mainly for the activity to review the literature to keep the knowledge update and use research results to improve practice. The problem based learning and the traditional educational method that were outcomes in moderate but significant differences. Similarly in our study, maximum number physical therapy students, agreed that scientific research exploring the effectiveness of physiotherapy treatment is necessary for positive growth in the physiotherapy profession less knowledge about physical therapy education and profession. Few of the students believed that scientific research exploring the cost versus benefits ratio of physiotherapy treatment is necessary for positive growth in the physiotherapy profession. Weber ${ }^{20}$ reported that students who replied ninety nine percent established that research was essential for positive development. 58\% intended to involve in research programs proceeding to graduation, a group of participants had research experience. Most of students showed technical writing challenging aspect of research, heavy academic burden highest restrictive to participation. Students were aware requirements for research and were showed interest in conducting research.

Whereas our study is comparatively more similar to JanaudisFerreira et al. ${ }^{21}$ reported that majority of the participants have been reported scientific research exploring the safety of physiotherapy treatment is necessary for positive growth in the physiotherapy profession. Participants response were as followed: 75\% (75) participants replied strongly agreed, 24\% (24) individual replied agreed and only $1(1 \%)$ individuals showed neutral response. Our study is also similar to Aljadi et al..$^{22}$ reported that mostly students believed scientific research will result in greater validation of physiotherapy treatment among the health care professions. Finally this study provides comprehensive detail of the barriers faced by students to participate in research activities which should be minimized in order to enhance the growth of the physical therapy profession.

\section{Conclusion}

Majority of the students perceive that research in undergraduate is necessary for positive growth of the physical therapy profession. They do participate in research activities but not all because of the common barriers faced by them in research related activities such as more workload and lack of time but they do believe that scientific research in postgraduate is necessary for positive growth in the physiotherapy profession.

\section{Acknowledgements}

None.

\section{Conflicts of interest}

Authors declare that there is no conflict of interest.

\section{References}

1. American Physical Therapy Association. Vision 2020: vision sentence for physical therapy and vision statement for physical therapy; 2020.

2. Kamwendo K. What do Swedish physiotherapists feel about research? A survey of perceptions, attitudes, intentions and engagement. Physiother Res Int. 2002;7(1):23-34.

3. Khan H, Khawaja MR, Waheed A, et al. Knowledge and attitudes about health research amongst a group of Pakistani medical students. BMC Med Educ. 2006;6(54).

4. Aslam F, Shakir M, Qayyum MA. Why Medical Students Are Crucial to the Future of Research in South Asia. PLoS Med. 2005;2(11):e322.

5. Sabzwari S, Kauser S, Khuwaja AK. Experiences, attitudes and barriers towards research amongst junior faculty of Pakistani medical universities. BMC Med Educ. 2009;9(68).

6. Vujaklija A, Hren D, Sambunjak D, et al. Can teaching research methodology influence students' attitude toward science? Cohort study and non-randomized trial in a single medical school. $J$ Investig Med. 2010;58(2):282-286.

7. Bridges PH, Carter VM, Phillips T, et al. Competencies for 2020: Revalidation of the curricular competencies. Universal Journal of Educational Research. 2013;1(3):228-239.

8. Williams B, Brown T, Winship W. The Mismatch between Perceived and Preferred Expectations of Undergraduate Paramedic Students. Inter J Allied H Sci Pract. 2012;10(4).

9. Oyeyemi AY, Oyeyemi AL, Rufai AA, et al. Physiotherapy students perception of their teachers clinical teaching attributes. African J H Prof Edu. 2012; 4(1):4-9.

10. Al-Yousuf N. Clinical teachers evaluation. J Bahrain Med Soc. 2013;19.

11. Fluit CR, Bolhuis S, Grol R, et al. Assessing the quality of clinical teachers: a systematic review of content and quality of questionnaires for assessing clinical teachers. J Gen Intern Med. 2010;25(12):1337-1345.

12. Al-Mohaimeed AA, Khan NZ. Perceptions of Saudi medical students on the qualities of effective teachers. A cross sectional study. Saudi Med J. 2014;(35):183-188.

13. Bussieres AE, Al Zoubi F, Stuber K, et al. Evidence-based practice, research utilization, and knowledge translation in chiropractic: a scoping review. BMC Complementary and Alternative Medicine. 2016;16(216):1-15.

14. Andrews D, Fong G, Hackam D, et al. Guide to knowledge translation planning at CIHR: Integrated and end-of-grant approaches. Canada: The Canadian Institutes of Health Research (CIHR); 2015.

15. Aljadi SH, Alrowayeh HN, Alotaibi NM, et al. Research amongst Physical Therapists in the State of Kuwait: Participation, Perception, Attitude and Barriers. Med Princ Pract. 2013;22:561566.

16. Kamwendo K. Do occupational therapy and physiotherapy 
students care about research? A survey of perceptions and attitudes to research. Scand J Caring Sci. 2001;15(4):295-302.

17. Bendixen RM, Kreider CM. Review of Occupational Therapy Research in the Practice Area of Children and Youth. Am J Occup Ther. 2011;65(3):351-359.

18. Karlsson U. What do Swedish occupational therapists feel about research? Scand Journal of Occupational Therapy. A survey of perceptions, attitudes, intentions, and engagement. Journal Scandinavian Journal of Occupational Therapy. 2009;14(4):221229.

19. Siamian H, Mahmoudi R, Habibi F, et al. Students' Attitudes Towards Research at Mazandaran University of Medical Sciences in 2015. Mater Sociomed. 2016;28(6):468-472.
20. Weber LI. Chiropractic students and research: assessing the research culture at a North American chiropractic college. $J$ Chiropr Educ. 2010;24(1):35-45.

21. Janaudis-Ferreira T, Araujo T, Marie Romano J, et al. Perspectives of Canadian Final-Year Physiotherapy Students on Cardiorespiratory Physiotherapy as a Career Choice. Physiother Can. 2016;68(3):282-289.

22. Aljadi SH, Alrowayeh HN, Alotaibi NM, et al. Research amongst Physical Therapists in the State of Kuwait: Participation, Perception, Attitude and Barriers. Med Princ Pract. 2013;(22):561-566. 